\title{
r-Process From Jet Ejecta of Magnetorotational Core Collapse Supernovae
}

\author{
F.-K. Thielemann*, R. Käppeli, C. Winteler, A. Perego, M. Liebendörfer, N. \\ Nishimura, N. Vasset \\ Dept. of Physics, Univ. of Basel, 4056 Basel, Switzerland \\ E-mail: f-k.thielemann@unibas.ch
}

\section{A. Arcones}

Institut für Kernphysik, Technische Universität Darmstadt, D-64289 Darmstadt, Germany

E-mail: almudena.arcones@physik.tu-darmstadt.de

The search for a site of the strong/main r-process component (reproducing the 3rd r-process peak and elements beyond) in the early Galaxy is still open. Regular core collapse supernovae seem not to attain the required $Y_{e}$ /entropy conditions in their neutrino wind. Neutron star mergers provide strong neutron-rich (low entropy) conditions, which permit the production of the heaviest elements (even leading to fission-cycling), but there exist doubts that they can contribute at metallicities of $[\mathrm{Fe} / \mathrm{H}]=-3$. Their occurrance requires a binary system with two prior supernova explosions (ejecting their metals), before - delayed by an inspiral phase - the merger and ejection of neutron-rich matter takes place. This asks for sites providing similar conditions at the end of the evolution of massive single stars in the early evolution of the Galaxy. The observation of $10^{15}$ Gauss neutron stars (magnetars, a few percent of all neutron stars), indicates - via flux conservation - the collapse of a $10^{12} \mathrm{G}$ core. Such rare initial conditions of cores with high rotation rates and strong magnetic fields could materialize in fast rotators at low metallicities with low mass loss rates preventing the loss of angular momentum. We examine such magneto-rotationally driven supernovae as sources of r-process elements. On the basis of thermodynamic histories of tracer particles from a 3D magneto-hydrodynamical core-collapse supernova model we find a peak distribution of $Y_{e}$ in the ejecta of 0.17 , which permits to reproduce the second and third peak of the solar r-process pattern. This rare progenitor configuration, characterized by a high rotation rate and a large magnetic field, leads to the formation of bipolar jets and provides a natural site for the strong r-process observed in the early Galaxy. The latter is characterized by a large scatter in e.g. $[\mathrm{Eu} / \mathrm{Fe}]$, indicating a rare and powerful event which takes longer to obtain an average mean than for alpha-elements, ejected from all core collapse supernovae.

\section{International Symposium on Nuclei in the Cosmos,}

August 5-12, 2012

Cairns, Australia

\footnotetext{
${ }^{*}$ Speaker.
} 


\section{Introduction}

Neutron-capture processes like the s- and the r-process require a local source / production site of neutrons due to their mean lifetime of only $15 \mathrm{~min}$. Seed nuclei are necessary to act as starting points for a long reaction chain of neutron captures and beta-decays. In the s-process neutrons are created in $(\alpha, n)$-reactions during stellar He- and $\mathrm{C}$-burning. This leads to neutron densities, determining the timescale for neutron-capture, being on average longer than the betadecay half-lives of produced unstable isotopes. The result is the typical s-process path along the line of stability. r-process conditions, producing isotopes 10-15 units off stability, require higher neutron densities by orders of magnitude. These can only be attained in explosions with initially high neutron densities and a subsequent expansion of matter. $(\alpha, n)$-reactions, enhanced due to temperatures attained in a passing shock wave, could result in high neutron densities. In realistic stellar models, however, the available amount of ${ }^{22} \mathrm{Ne}$ or ${ }^{13} \mathrm{C}$ seemed not sufficient for this to occur (Truran et al. 1978, Thielemann et al. 1979, Cowan et al. 1985). Recent rotating stellar models, producing primary ${ }^{22} \mathrm{Ne}$ (Frischknecht et al. 2012), might change this. Other options include environments, where, due to very high densities, electrons are degenerate with high Fermi energies. The $\beta^{-}$-decay of neutrons is countered efficiently by electron captures, making neutrons abundant and "effectively" stable. Such conditions are attained in neutron stars with a high $\mathrm{n} / \mathrm{p}$ ratio of the order of 10 (i.e. a proton/nucleon ratio $Y_{e}=0.1$ ). The expansion of such neutron star-like matter would permit a strong r-process. Another option is the so-called very alpha-rich freeze-out from explosive Si-burning with a moderate neutron excess, i.e. $Y_{e} \approx 0.4-0.48$. Here the main effect is that initially very hot explosive conditions photodisintegrate all nuclei, leading to a composition of neutrons, protons and alphas. In the expansion and with decreasing temperatures
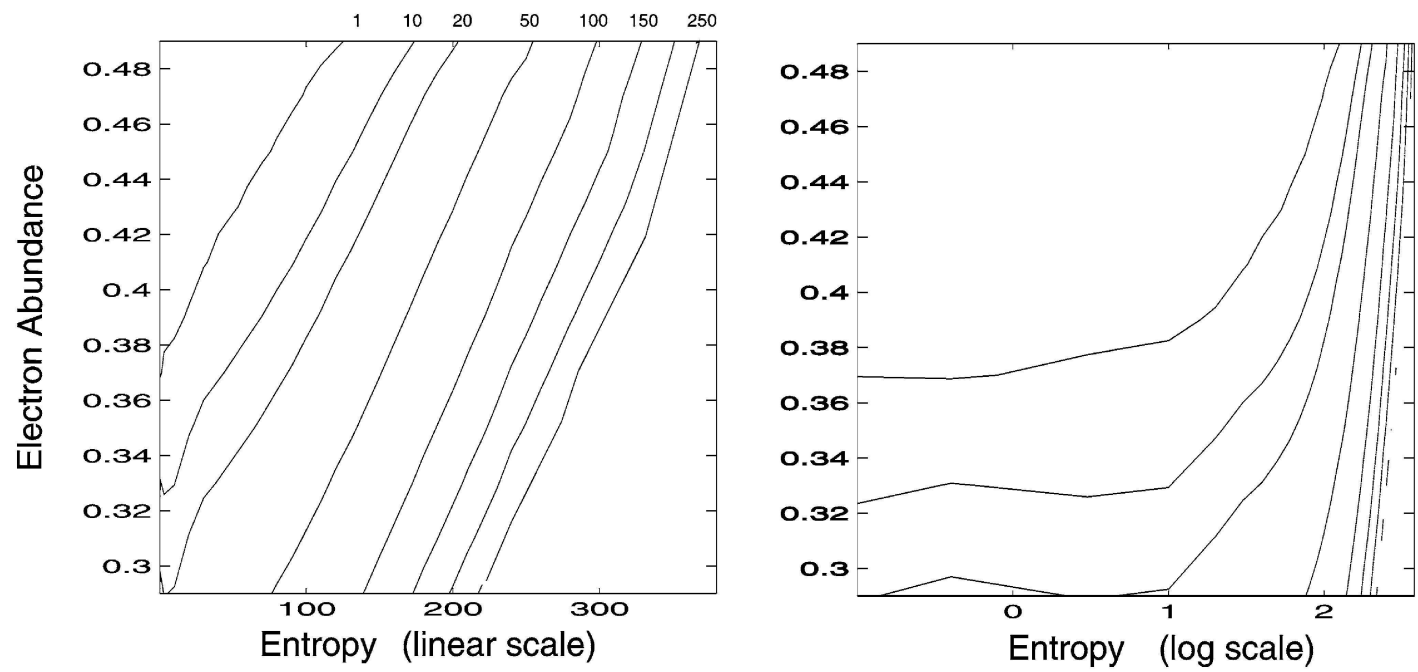

Figure 1: A full r-process requires $A_{\text {seed }}+n /$ seed $=A_{\text {actinide }}$, i.e. an $\mathrm{n} /$ seed ratio of at least 150 . This can be achieved for high entropies, measured in $\mathrm{k}_{B}$ /nucleon, with a moderate $Y_{e}$ (left) or for low entropies with a very low $Y_{e}$ (right). The lines indicate $\mathrm{n} / \mathrm{seed}$ ratios, the same for both figures. For low entropies these lines are only a function of $Y_{e}$ (right), a value of 150 is attained at $Y_{e} \approx 0.15$ (from Freiburghaus et al. 1999). 
matter tries to recombine to heavier nuclei. For high entropies (i.e. low $\rho / T^{3}$-ratios) matter is hindered to pass $\mathrm{He}$ (similar to the big bang) because reactions like $\alpha \alpha \alpha \rightarrow{ }^{12} \mathrm{C}$ or $\alpha \alpha n \rightarrow{ }^{9} \mathrm{Be}$, involving a highly unstable intermediate nucleus, are hindered at low densities and do not permit to build up Fe-group nuclei in the fast expansion. Then, if more than $90 \%$ of matter exists in the form of $\mathrm{N}=\mathrm{Z}$ alphas, the moderate neutron excess is sufficient to cause a high neutron/seed ratio in the remaining matter. The latter two options are displayed in Fig.1 for a typical hydrodynamic expansion timescale. They could stand for a neutrino wind in supernovae or expansions of highly compressed neutron-rich matter occurring in neutron star mergers or jet ejecta of supernovae.

Can observations of low metallicity stars give us a clue about the possible site? Eu is a typical r-process element, although being present in different amounts (a) in so-called Sneden stars which show the full heavy r-process pattern in solar proportions and (b) Honda-type stars which do show Eu but no r-process elements of the third peak and beyond (and Eu to first r-process peak ratios also smaller than in solar proportions). Fig.2 displays $[\mathrm{Eu} / \mathrm{Fe}]$ observations as a function of metallicity. The average $[\mathrm{Eu} / \mathrm{Fe}]$ indicates a typical core collapse supernova behavior, as also found e.g. in all alpha elements $[\alpha / \mathrm{Fe}]$. The huge scatter by more than two orders of magnitude, before showing this typical average behavior, indicates (a) a rare but powerful event, and possibly (b) a mixture of two types of events, where the Sneden-type stars dominate the overall production during galactic evolution, as they show the solar pattern. Neutron star mergers can match both criteria, being rare but producing sufficient amounts of heavy r-process elements in solar proportions. There exist, however, doubts that their products would already be visible at metallicities of $[\mathrm{Fe} / \mathrm{H}]=-3$ (Argast et al. 2004). Some recent studies, which include the fact that our Galaxy is possibly the result from smaller merging subsystems (with different star formation rates) have been expected to show a way out of this dilemma (Ishimaru \& Wanajo 2010). If this cannot be solved, we need another strong r-process source already at low metallicities, and possibly jets from rotating core collapses with strong magnetic fields could be the solution (Cameron 2003; Nishimura et al. 2006; Fujimoto et al. 2008). In the following we want to present the case for r-process jets of highly compressed neutronrich matter (with similar conditions and abundance results as in neutron star mergers), originating from a rare subset of core collapse supernovae with high rotation rates and strong magnetic fields, which could provide the observed pattern already at lowest metallicities.

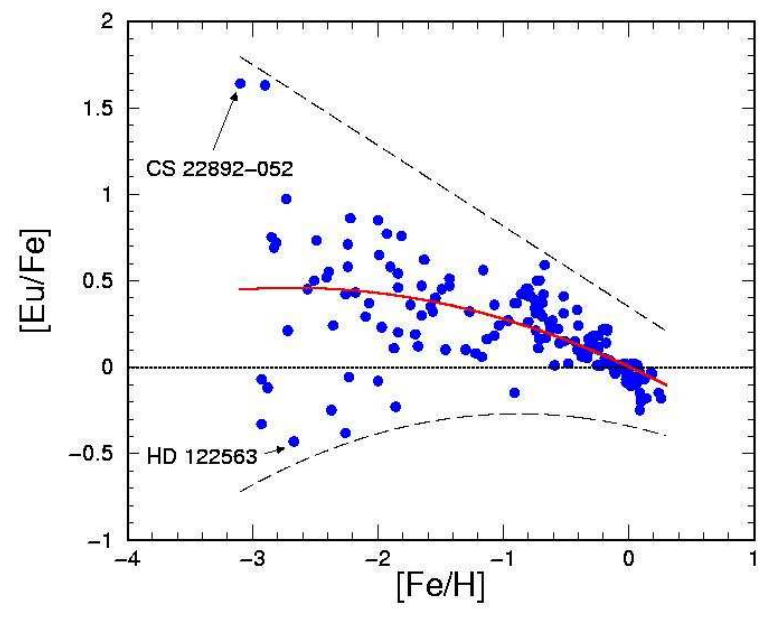

Figure 2: Observed $[\mathrm{Eu} / \mathrm{Fe}]$ ratios at low metallicities (Cowan \& Thielemann 2004). Visible is the large scatter before the average pattern is attained (shown as a red line). The plot shows also entries from Snedentype stars with a relative solar pattern for the heavy r-elements (CS 22892-052) and Honda-type stars with a missing third rprocess peak and non-solar patterns also for the other r-elements (HD 122563). Apparently, no star not showing an r-process contribution is observed. 


\section{Supernova Models with fast Rotation and high Magnetic Fields}

The supernova ( $\mathrm{SN})$ mechanism, explaing how massive stars end in a central collapse to a neutron star and explosive ejection of the outer layers, is still debated. It has been related to neutrino emission from the hot collapsed core and accreted matter (Bethe 1990). Recent multi-D hydrodynamic approaches and improved (spectral) neutrino transport (Müller et al. 2010; Lentz et al. 2012; Liebendörfer et al. 2010; Burrows et al. 2011) give an optimistic outlook. An equation of state (EoS) caused explosion scenario has found a recent revival, based on the quark-hadron phase transition at supranuclear densities (Sagert et al. 2009), but its working depends on choices of EoS properties in a narrow parameter range. Fast rotation and strong magnetic fields have been discussed for more than 30 years, but required 3D modeling. The major outcome was that high rotation rates and (possibly unrealistically high) magnetic fields were required to launch explosions. The question is whether such magnetic fields can be attained during collapse with rotation and on which timescale after collapse (LeBlanc \& Wilson 1970; Meier et al. 1976; Bisnovatyi-Kogan et al. 1976; Müller \& Hillebrandt 1979). This topic has recently been re-addressed by Moiseenko \& Bisnovatyi-Kogan (2008) and Takiwaki et al. (2009).

The results of our 3D magneto-hydrodynamics investigations are obtained with the code FISH (Käppeli et al. 2011). They are discussed in more detail in Winteler et al. (2012). The gravitational potential is approximated by an effective axisymmetric mass distribution that includes general relativistic monopole corrections (Marek et al. 2006), and the Lattimer \& Swesty (1991) equation of state (EoS) with nuclear compressibility $180 \mathrm{MeV}$ was utilized. Tracer particles, acting
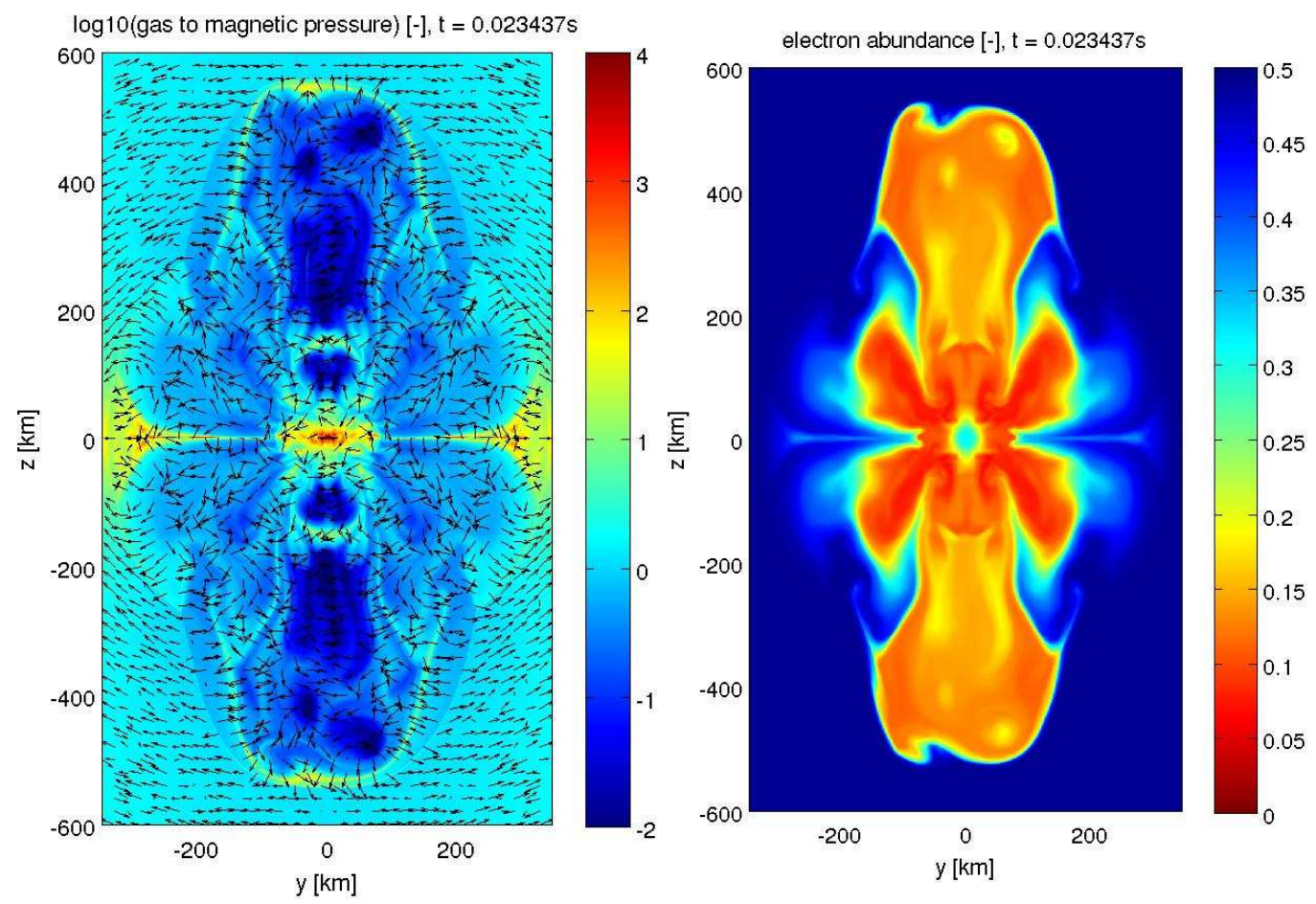

Figure 3: Contourplots for two physical quantities $21 \mathrm{~ms}$ after bounce. Color scales displayed on right side of both plots. Left: Ratio of gas pressure and magnetic pressure $\left(B^{2} / 8 \pi\right)$. Right: Geometrical $Y_{e}$-distribution. 


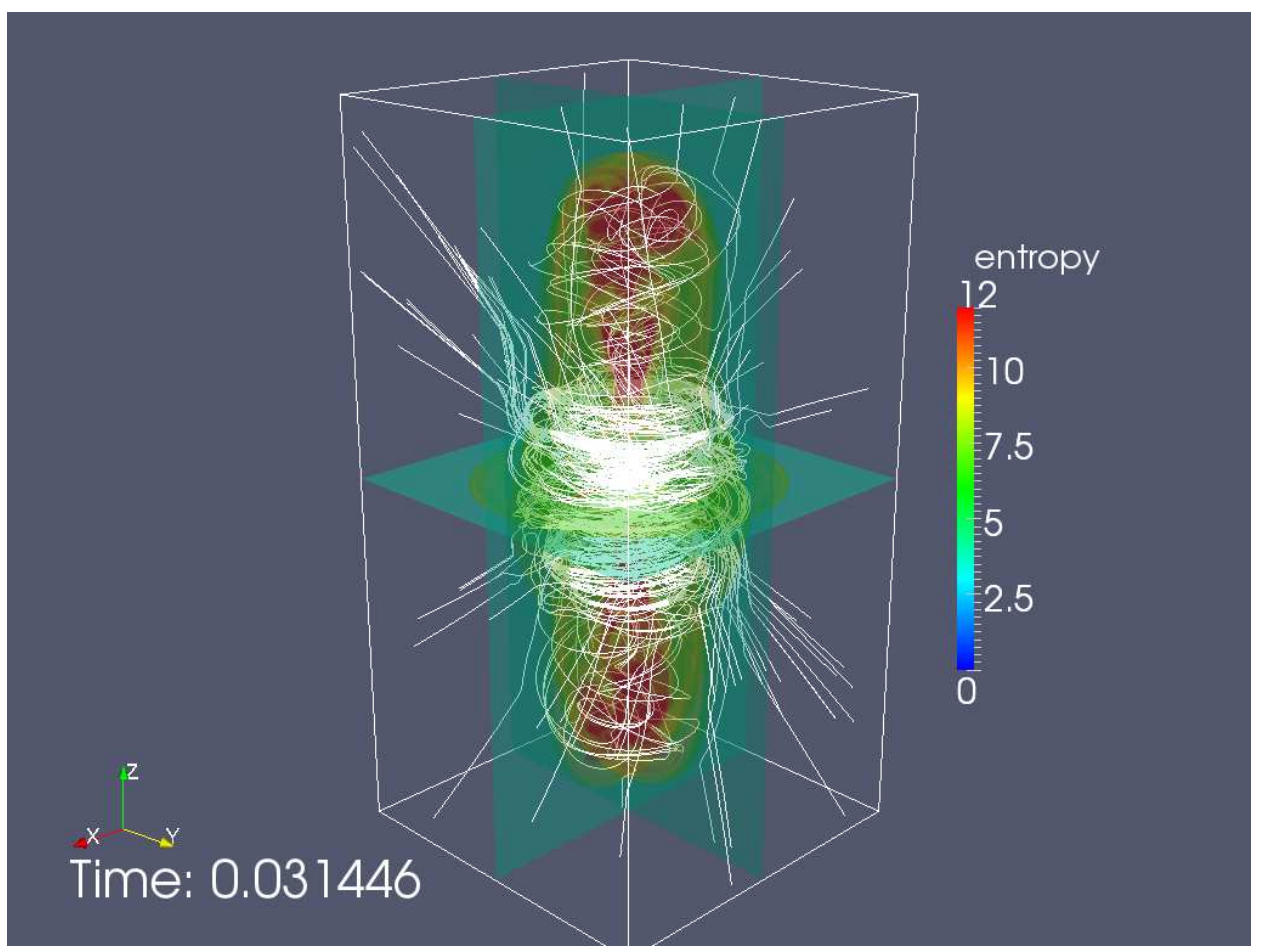

Figure 4: The 3D domain size $(700 \times 700 \times 1400)$ of the simulation $31 \mathrm{~ms}$ after bounce, showing entropy contours on a color scale. Magnetic field lines are shown in white, indicating a strong winding of the magnetic field.

as a Lagrangian component, are passively advected with the flow and record the thermodynamic conditions of a particular fluid element for the post-processing nucleosynthesis calculations. The transport of the electron neutrinos and anti-neutrinos is treated by a 3D spectral leakage scheme, based on previous grey leakage schemes (Rosswog \& Liebendörfer 2003; Ruffert et al. 1997) but here making use of 12 energy groups spanning the range $E_{v}=3-200 \mathrm{MeV}$. All fundamental neutrino reactions have been included, providing detailed spectral emissivities and opacities (Bruenn 1985). Inside the neutrinosphere, weak equilibrium is assumed and trapped neutrinos are modeled accordingly; outside of it, no explicit absorption is considered. Thus we can only follow neutrino emission and the associated neutronization of matter. Burrows et al. (2007) has shown, that neutrino heating contributes only $10-25 \%$ to the explosion energy and is therefore less important. This justifies our pragmatic approach. We employed the pre-collapse $15 \mathrm{M}_{\odot}$ model of Heger et al. (2005). An initial shellular rotation law was assumed $\Omega(r)=\Omega_{0} R_{0}^{2} /\left(r^{2}+R_{0}^{2}\right), \Omega_{0}=\pi s^{-1}$ and $R_{0}=1000$. The magnetic field was approximated by a homogeneous, purely poloidal field of $5 \times 10^{12} \mathrm{G}$. Gravitational collapse occurs until core-bounce, the conservation of angular momentum in combination with the collapse leads to a massive spin-up of the core and significant rotationally induced deformations. The magnetic field is amplified by magnetic flux conservation up to $\approx 5 \times 10^{15} \mathrm{G}$. After bounce, differential rotation winds up the poloidal field into a very strong toroidal field, increasing the magnetic energy/pressure at the expense of rotational energy. Strongly magnetized regions appear near the rotational axis with an associated magnetic pressure quickly reaching and exceeding that of the local gas pressure. The Lorentz force then becomes dynamically important and drives a bipolar 
outflow, i.e. jets. The jets propagate rapidly along the rotational axis. Fig.4 displays a snapshot at $31 \mathrm{~ms}$.

\section{Nucleosynthesis in Jet Ejecta}

The nucleosynthesis calculations were performed with our new extended reaction network (Winteler 2011) which represents an advanced (numerically and physically) update of the BasNet network (see e.g. Thielemann et al. (2011)) with weak interaction rates as in Arcones \& MartinezPinedo (2011). We include neutron capture, induced fission and beta-delayed fission rates from Panov et al. (2010) and Panov et al. (2005). The tracer particles obtained from the simulation provide density, temperature, and electron fraction for the nuclear network, as well as position and velocity, from the beginning to the end of the simulation $\left(t=t_{f}\right)$. After $t_{f}$, thermodynamic variables are evolved following the prescription in Fujimoto et al. (2008). For the post-processing we only consider gravitationally unbound tracer particles. The electron fraction is a key input for the nucleosynthesis and strongly depends on details of the neutrino transport. Although neutrino absorption is crucial to determine the $Y_{e}$, only the leakage scheme was included in the hydrodynamical simulations (where it is expected to have a minor impact on the dynamics). Therefore, we present two different nucleosynthesis calculations: (1) $Y_{e}$ is taken from the original tracer particles, (2) the effect of neutrino absorption on $Y_{e}$ is included, utilizing the neutrino information obtained from the leakage scheme in order to update the electron fraction outside the neutrinosphere. The electron fraction of the tracer is evolved using approximated rates for the neutrino emission and absorption on nucleons (see Janka 2001). We also consider neutrino reactions from Fröhlich et al. (2006) in the nucleosynthesis network. Fig. 5a shows the ejected mass as a function of $Y_{e}$ for the original simulation data and for the case including neutrino absorption. These corrections shift the peak distribution from $\approx 0.15$ to 0.17 and broaden it towards higher $Y_{e}$. In both approaches, at the onset of the nucleosynthesis, the density is still relatively high, $\rho \approx 10^{9} \mathrm{gcm}^{3}$, and the electron fraction rather low, $Y_{e} \approx 0.15-0.3$, providing conditions close to those in neutron star mergers.

Mass integrated abundances are presented in Fig. 5b. Nuclei around the second $(A=130)$ and third $(A=195)$ r-process peaks, and up to the actinides, can be synthesized in the jets and the position of the peaks nicely agrees with solar system abundances. The large trough in the mass range 140-160 is due to the strong $\mathrm{N}=82$ shell-closure of the FRDM mass model and a simplified prescription of fission fragments. Nuclei in the region below the second peak are affected by the $Y_{e}$ distribution, where higher $Y_{e}$ 's cause a smaller $\mathrm{n} /$ seed ratio, leading to larger abundances in the range $A=80-100$ and a less abundant third peak and the higher mass region.

\section{Conclusions}

Magneto-rotationally driven supernovae suffer one main critique: the simultaneous presence of fast rotation and strong magnetic fields in the progenitor before collapse. Recent progenitor models computed by Heger et al. (2005) suggest that these special conditions are not reached in common evolutionary paths of massive stars. However, the low-metallicity models (Woosley \& Heger 2006, Hirschi 2007)) indicate that for a fraction, perhaps $1 \%$, of all massive stars, favorable conditions can appear under special circumstances. This rarity of progenitors with these special 

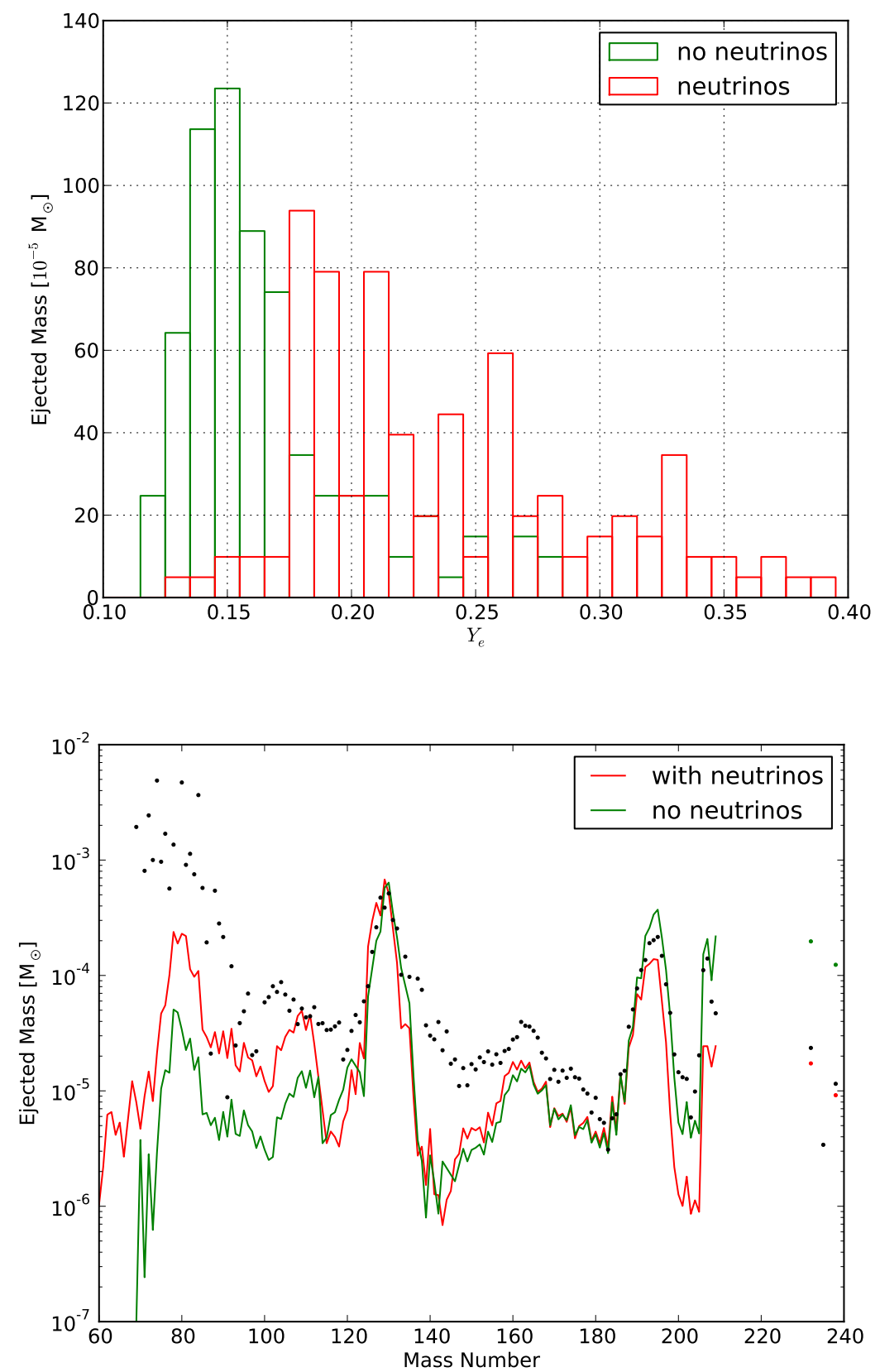

Figure 5: Top: Ejected mass vs. $Y_{e}$ for the original simulation without neutrino captures (green) and including a simplified prescription for neutrino heating (red). Bottom: Integrated mass fractions for nucleosynthesis calculations with (red) and without (green) neutrino heating. Black dots represent solar r-process abundances (Sneden et al. 2008) scaled to fit the red line at $\mathrm{A}=130$.

initial conditions can also be put into accordance with the observed scatter of r-process element abundances at low metallicity, combined with the high r-process production of $10^{-2} \mathrm{M}_{\odot}$ per event. This indicates that these elements have been produced in relatively scarce but efficient events. 
Therefore, the rare progenitor configuration used here could naturally provide a strong r-process site in agreement with observations of early galactic chemical evolution. Currently, long-term global 3D simulations of core-collapse supernovae with sophisticated neutrino transport and the aim to simulate local flow instabilities for magnetic field amplification, seem to be mutually exclusive (Obergaulinger et al. 2009). Therefore, we started with the assumption of sufficiently strong initial magnetic fields, relying on a physical process that can quickly amplify the magnetic field to dynamic importance. This, plus the existence of $10{ }^{15} \mathrm{G}$ neutron stars (magnetars: Kouveliotou et al. 1998; Kramer 2009), motivates our choice of the high initial poloidal field strength, which by flux compression and rotational winding leads to magnetic fields whose magnitude roughly agree with those expected from the magnetorotational instability at saturation (Obergaulinger et al. 2009). The main results of this investigation is that full 3D calculations can support the emergence of bipolar jets and that these are not artifacts of up to now axissymmetric approaches (Nishimura et al. 2006, Fujimoto et al. 2008). Such explosions, resulting from the individual evolution of massive stars rather than complicated binary histories of neutron star mergers, could explain strong r-process features during early galactic evolution, as observed in low metallicity stars. The r-process production is very efficient, a factor of 100 more than required on average from supernovae, permitting that only about $1 \%$ undergo such events.

\section{References}

[1] Arcones, A., Martinez-Pinedo, G., Phys. Rev. C 83 (2011) 045809

[2] Argast, D., Samland, M., Thielemann, F.-K., Qian, Y.-Z., A\&A 416 (2004) 997

[3] Bisnovatyi-Kogan, G. S., Popov, Y. P., Samochin, A. A., Ap\&SS 41 (1976) 321

[4] Burrows, A., Dessart, L., Livne, E., Ott, C. D., Murphy, J., ApJ 664 (2007) 416

[5] Burrows, A.S., Nordhaus, J., Almgren, A, Bell, J., Com. Phys. Comm. 182 (2011) 1764

[6] Cameron, A. G. W., ApJ 587 (2003) 327

[7] Cowan, J.J., Cameron, A.G.W., Truran, J.W., ApJ 294 (1985) 656

[8] Cowan, J.J., Thielemann, F.-K., Phys. Today 57 (2004) 47

[9] Freiburghaus, C. et al., ApJ 516 (1999) 381

[10] Frischknecht, U., Hirschi, R., Thielemann, F.-K., A\&A 538 (2012) L2

[11] Fröhlich, C., et al., Phys. Rev. Lett. 96 (2006) 142502

[12] Fujimoto, S., Nishimura, N., Hashimoto, M., ApJ 680 (2008) 1350

[13] Heger, A., Woosley, S. E., \& Spruit, H. C., ApJ 626 (2005) 350

[14] Hirschi, R., ApJ 461 (2007) 571

[15] Honda, S., Aoki, W., Ishimaru, Y., Wanajo, S. 2007, ApJ 666 (2007) 1189

[16] Ishimaru, Y., Wanajo, S. (2010), privare communication

[17] Janka, H.-T., A\&A 368 (2001) 527

[18] Käppeli, R., Whitehouse, S. C., Scheidegger, S., Pen, U.-L., \& Liebendörfer, M., ApJS 195 (2011) 20 
[19] Kouveliotou, C., et al., Nature 393 (1998) 235

[20] Kramer, M., IAU Symposium 259 (2009) 485

[21] Lattimer, J. M., \& Swesty, F. D., Nucl. Phys. A 535 (1991) 331

[22] LeBlanc, J. M., Wilson, J. R., ApJ 161 (1970) 541

[23] Lentz, E. J., et al., ApJ 747 (2012) 73

[24] Liebendörfer, M., et al., Prog. Theor. Phys 186 (2010) 87

[25] Marek, A., Dimmelmeier, H., Janka, H.-T., Müller, E., \& Buras, R., A\&A 445 (2006) 273

[26] Meier, D. L., et al., ApJ 204 (1976) 869

[27] Moiseenko, S. G., Bisnovatyi-Kogan, G. S., Int. J. Mod. Phys. D 17 (2008) 1411

[28] Müller, B., Janka, H.-T., Dimmelmeier, H., ApJS 189 (2010) 104

[29] Müller, E., Hillebrandt, W., A\&A 80 (1979) 147

[30] Nishimura, S., et al., ApJ 642 (2006) 410

[31] Obergaulinger, M., et al., $A \& A 498$ (2009) 241

[32] Panov, I. V., et al., Nucl. Phys. A 747 (2005) 633

[33] Panov, I. V., et al., A\&A 513 (2010) A61

[34] Rosswog, S., \& Liebendörfer, M., Mon. Not. Roy. Astron. Soc. 342 (2003) 673

[35] Ruffert, M., Janka, H.-T., Takahashi, K., \& Schaefer, G., A\&A 319 (1997) 122

[36] Sagert, I. et al., Phys. Rev. Lett. 192 (2009) 081101

[37] Sneden, C., Cowan, J.J., Gallino, R., ARA\&A 46 (2008) 241

[38] Takiwaki, T., Kotake, K., Sato, K., ApJ 691 (2009) 1360

[39] Thielemann, F.-K., Arnould, M., Hillebrandt, W., A\&A 74 (1979) 175

[40] Thielemann, F.-K., et al., Prog. Part. Nucl. Phys. 66 (2011) 346

[41] Truran, J.W., Cowan, J.J., Cameron, A.G.W., ApJ 222 (1978) L63

[42] Winteler, C., Käppeli, R., Perego, A., Arcones, A., Vasset, N., Nishimura, N., Liebendörfer, M., Thielemann, F.-K., ApJ 750 (2012) L22

[43] Woosley, S. E., Heger, A., ApJ 637 (2006) 914

\section{Questions:}

Francesca Matteucci: How long is the delay time between the formation of the neutron stars their merging? Answer: The binary pulsar has a $10^{8} \mathrm{y}$ delay, initial configurations with higher ellipticity could come down to $10^{6} \mathrm{y}$.

Alex Heger: We have never seen a star with no r-process. Why is this not inconsistent with having rare events making the r-process? Answer: Seeing Eu does not mean that a strong r-process with production of the 3rd peak occurred. Honda-type abundance patterns (which also include Eu) could possibly originate from regular supernova ejecta, if the new correct treatment of in-medium potentials of neutrons and protons supports such results. 